$17.1 \mid 2013$

Varia

\title{
Ancienne abbaye de Saint-Gilles-du-Gard. Nouvelles recherches sur la sculpture architecturale « erratique »
}

Andreas Hartmann-Virnich et Marie-Pierre Bonetti

\section{(2) OpenEdition}

Journals

Édition électronique

URL : https://journals.openedition.org/cem/13004

DOI : $10.4000 /$ cem. 13004

ISSN : 1954-3093

Éditeur

Centre d'études médiévales Saint-Germain d'Auxerre

Référence électronique

Andreas Hartmann-Virnich et Marie-Pierre Bonetti, « Ancienne abbaye de Saint-Gilles-du-Gard Nouvelles recherches sur la sculpture architecturale « erratique » », Bulletin du centre d'études médiévales d'Auxerre | BUCEMA [En ligne], 17.1 | 2013, mis en ligne le 03 juin 2013, consulté le 22 septembre 2022. URL : http://journals.openedition.org/cem/13004 ; DOl : https://doi.org/10.4000/ cem. 13004

Ce document a été généré automatiquement le 22 septembre 2022.

\section{(c) (i) $(9)$}

Creative Commons - Attribution - Pas d'Utilisation Commerciale - Partage dans les Mêmes Conditions 4.0 International - CC BY-NC-SA 4.0

https://creativecommons.org/licenses/by-nc-sa/4.0/ 


\title{
Ancienne abbaye de Saint-Gilles-du- Gard. Nouvelles recherches sur la sculpture architecturale « erratique »
}

\author{
Andreas Hartmann-Virnich et Marie-Pierre Bonetti
}

1 Les fouilles archéologiques dans l'ancienne aire claustrale de l'abbaye de Saint-Gilles, effectuées dans le cadre du programme pluridisciplinaire ANR-DFG AEGIDIANA (2009-2010) ${ }^{1}$, ont permis d'identifier les traces matérielles des

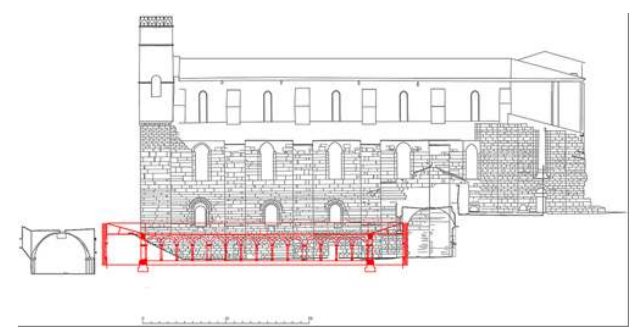
galeries du cloître roman, jusqu'alors absentes, et des fragments inédits de son décor sculpté. Le démontage complet des murs-bahuts, vers la seconde moitié du xvIII ${ }^{e}$ siècle, n'avait en effet laissé que des tranchées d'épierrement, comblées et scellées par une couche de cendres qui pourrait provenir de l'installation éphémère d'un four à chaux, qui n'a toutefois pas été découvert sur l'emprise de la fouille. 
Fig. 1 - Éléments architecturaux découverts en fouille dans le comblement d'une tranchée (cl. L. Buffat).

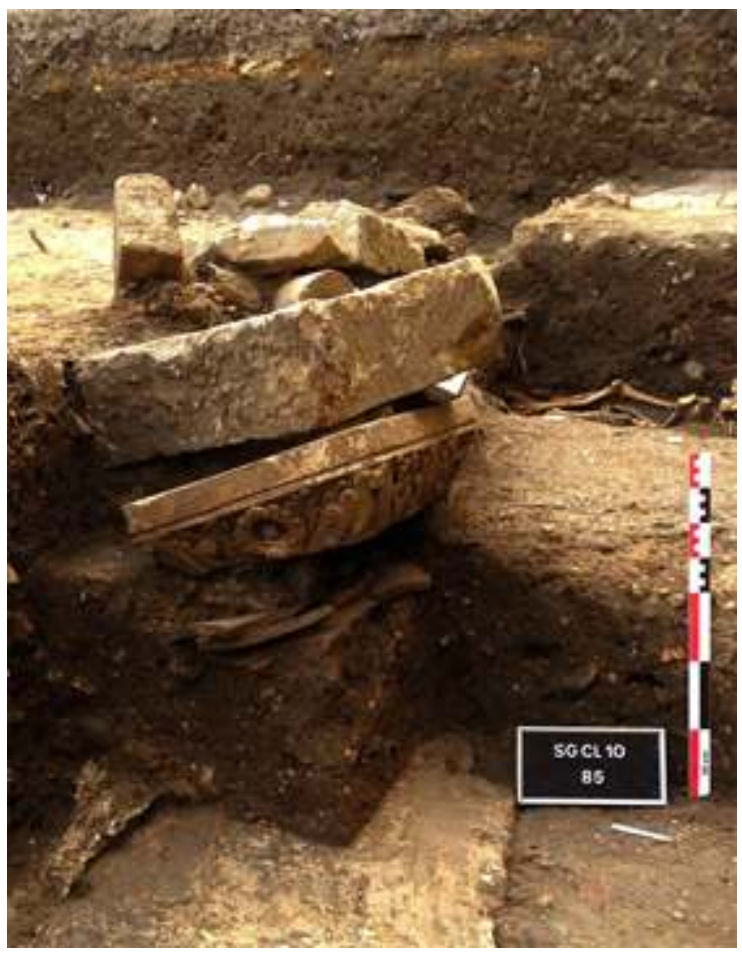

Le dépôt lapidaire empilé dans le comblement d'une tranchée de récupération connexe, qui avait suivi le tracé d'un conduit maçonné incorporé dans les fondations du murbahut nord (fig. 1), se composait de plusieurs fragments architecturaux provenant selon toute probabilité des arcades de la galerie contiguë du cloître : un tailloir intact, plusieurs fragments de fûts de colonnettes d'un même gabarit, de bases, de feuilles d'acanthe, d'abaque et de volute de chapiteaux de type corinthien (fig. 2), un sommier, un claveau et un écoinçon d'arcade, plusieurs fragments de sculpture figurative en haut-relief décorant des motifs architecturaux miniaturisés, tels que pilastres cannelés et arcades, ainsi qu'un fragment d'une inscription datable de la fin du XIII ${ }^{e}$ ou du début du XIV ${ }^{\mathrm{e}}$ siècle ${ }^{2}$.

Fig. 2 - Fragment d'un chapiteau à feuilles d'acanthe issu des fouilles (cl. M.-P. Bonetti).

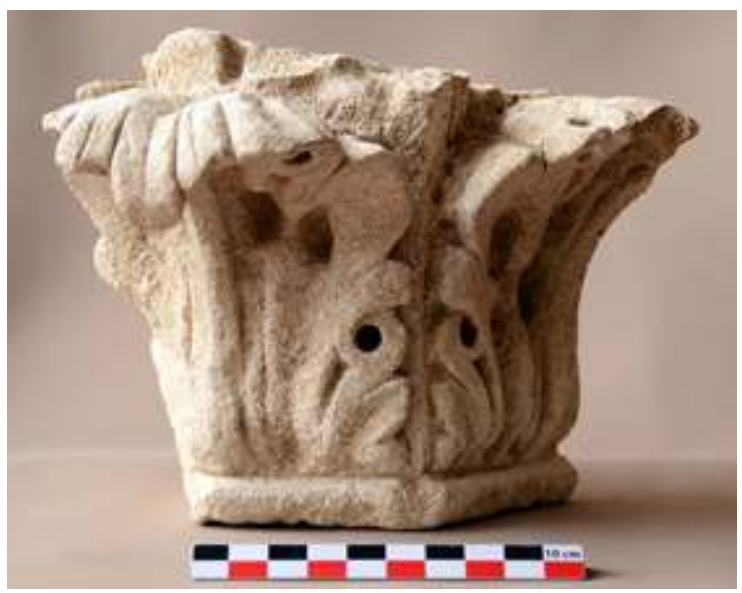


3 La découverte de ces fragments revêt une importance toute particulière dans la mesure où il s'agit des seuls éléments de cette partie du monument issus d'un contexte archéologique connu et stratifié. Leur étude est, de ce fait, capitale non seulement pour une enquête élargie, prenant en compte les éléments épars aujourd'hui conservés dans les collections du musée ou déposés dans la crypte de l'abbatiale, mais aussi pour l'analyse stylistique du décor du cloitre, dont l'antériorité à l'abbatiale, confirmée par les recherches archéologiques récentes ${ }^{3}$, suppose une reconstruction de la galerie nord au sud de son emplacement initial, sous le bas-côté de la nouvelle église, beaucoup plus large que l'envergure présumée de son prédécesseur. En effet, la similitude stylistique des fragments retrouvés avec la sculpture de la façade et des parties contemporaines du chevet, tout comme l'analyse du parement sud de l'abbatiale, suggèrent que la reconstruction de la galerie nord fut entreprise en même temps.

4 Si dans un premier temps un catalogue descriptif des pièces découvertes a bien été dressé ${ }^{4}$, l'étude archéologique de ce mobilier lapidaire, particulièrement fragmentaire, a donc nécessité une réévaluation détaillée des éléments de sculpture erratique présents dans le complexe abbatial, et de ceux attribués à ce dernier conservés au musée de la Maison romane de Saint-Gilles, qui présentent clairement des caractéristiques similaires de par leur nature et leur style.

5 Toutefois, l'exigence d'outrepasser l'apparence des formes, pour soumettre ces vestiges sculptés à une analyse de fond afin de pouvoir, à terme, les mettre en relation avec la sculpture architecturale in situ de l'abbatiale, incitait à adopter de nouveaux regards archéologiques sur ces documents historiques et la mise au point d'une nouvelle documentation de leurs caractéristiques techniques et stylistiques.

6 En effet, une nouvelle réflexion, méthodologique et pluridisciplinaire, s'est avérée nécessaire pour étudier la sculpture architecturale d'un édifice aussi complexe que l'abbaye de Saint-Gilles-du-Gard, en écartant l'approche traditionnelle, aussi subjective qu'inopérante et infructueuse ${ }^{5}$, de l'attribution des éléments sculptés à d'hypothétiques «maîtres» et «ateliers ». Par ailleurs, un réexamen de la sculpture architecturale apportera certainement des indices complémentaires à l'archéologie du bâti et du sous-sol pour la datation de l'édifice et la restitution de la chronologie relative et absolue du chantier. Qu'il s'agisse des éléments découverts en fouille ou des fragments erratiques regroupés dans le dépôt lapidaire, l'étude de la sculpture architecturale saint-gilloise renseigne sur le travail des tailleurs de pierre, les outils employés, les techniques maîtrisées, les modèles utilisés et l'organisation générale du chantier abbatial.

7 Ainsi, l'objectif principal du travail de recherche entrepris dans le cadre d'un master en archéologie et histoire de l'art de l'université d'Aix-Marseille, au sein du Laboratoire d'archéologie médiévale et moderne en Méditerranée (LA3M - UMR 7298), est donc de fournir un éclairage nouveau sur la sculpture architecturale ex situ de l'abbatiale. Largement encouragé et soutenu institutionnellement par la ville de Saint-Gilles-duGard, en particulier la responsable du musée et du patrimoine, Vanessa Eggert, ce travail participe au projet collectif de recherche AEGIMAIOR (2012-2015) ${ }^{6}$ et bénéficie, cette année, d'une allocation de formation et de recherche attribuée au titre des Monuments historiques par la Direction générale des patrimoines du ministère de la Culture et de la Communication.

8 Cette étude entend placer et affiner la chronologie relative de la sculpture saint-gilloise par rapport aux différentes phases de construction de l'abbatiale et à l'évolution des 
bâtiments claustraux. De premières hypothèses de reconstruction du cloître et de son décor sculpté, basées sur des critères objectifs, ont été formulées, notamment pour la galerie nord, nécessairement plus récente que les autres galeries déjà en place, dont les parties non touchées par le chantier de la grande abbatiale durent échapper au démontage.

De même, une hypothèse de recomposition du décor sculpté du chœur détruit a ensuite été envisagée à partir de l'étude approfondie des fragments conservés in situ et au musée municipal, tandis qu'une analyse de la sculpture saint-gilloise, comprise dans son contexte artistique local et régional, a été entamée, en prêtant une attention particulière aux modes de transmission des modèles.

Pour ce faire, un protocole d'étude multidisciplinaire de la sculpture, en tant qu'objet archéologique, a été mis en place pour réduire la subjectivité des critères d'analyse et de datation. Pensé pour fournir des outils scientifiques sur lesquels développer une interprétation stylistique, il comporte plusieurs étapes.

11 Une première étape a consisté dans la création d'une base de données qui répertorie la totalité de la sculpture architecturale ex situ provenant du site. Elle se compose de trois inventaires correspondant respectivement aux éléments conservés au musée ${ }^{7}$, à ceux mis au jour lors de la fouille et aux pièces erratiques dispersées entre l'église basse, l'ancien chœur, les bâtiments claustraux et les maçonneries postmédiévales. Le travail d'analyse facilité par cette base de données a d'ores et déjà permis de sélectionner des éléments pour réaliser une étude de cas. Celle-ci porte sur les chapiteaux conservés, qui pourraient, de par leurs dimensions, provenir du cloître de l'abbatiale (fig. 3).

Fig. 3 - Chapiteau à feuilles d'acanthe et protomé du musée de la Maison romane (cl. M.P. Bonetti).

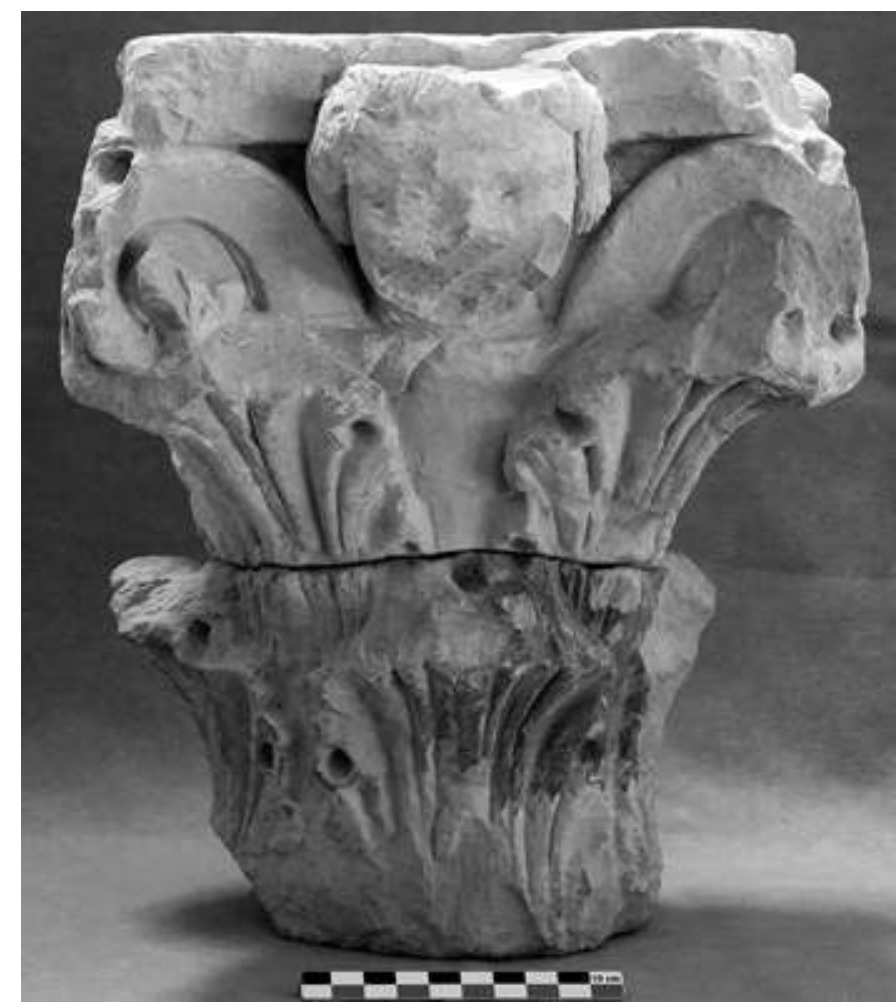



sculptés en utilisant le relevé manuel comme protocole d'examen technique et outil d'analyse privilégié (fig. 4).

Fig. 4. - Relevés manuels de chapiteaux conservés au musée de la Maison romane (M.-P. Bonetti).

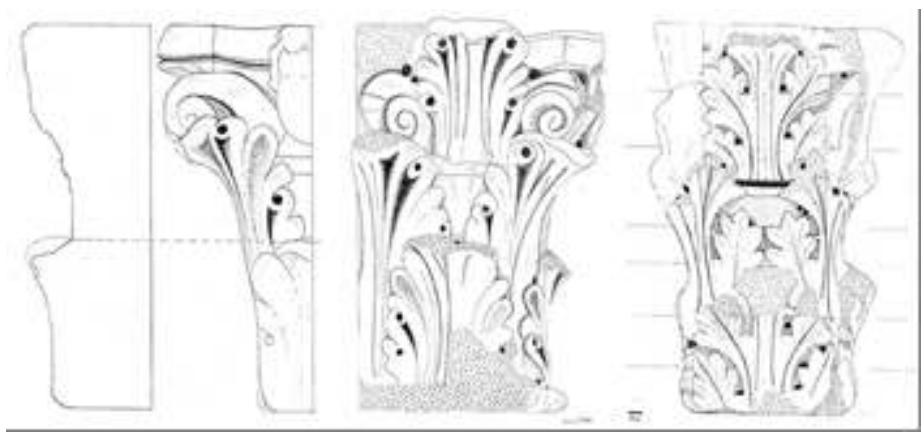

13 Ce dernier est accompagné de relevés photogrammétriques afin d'évaluer les potentialités offertes par les nouvelles technologies numériques en phase d'étude de la sculpture, bien en amont du travail de valorisation culturelle ou de divulgation scientifique et pédagogique.

Fig. 5 - Modélisation par photogrammétrie 3D du tailloir découvert en fouille (CAO M.-P. Bonetti).

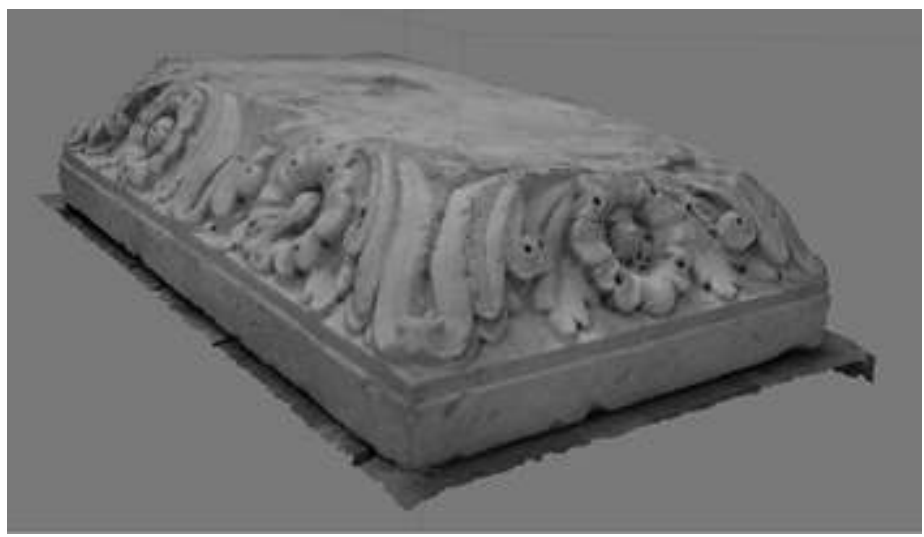

En effet, les modèles tridimensionnels générés (fig. 5) doivent servir tant à l'anastylose virtuelle d'une galerie du cloître, qu'à l'analyse de la conception des chapiteaux. Ce travail, encore en cours, qui s'intéresse aux tracés d'épures, à l'épannelage, mais aussi au façonnage et à la sculpture des détails, s'inspire directement de la rétro-ingénierie.

Enfin, une troisième et dernière étape vise à une interprétation stylistique comparative basée sur les données scientifiquement collectées, pour établir une cartographie chrono-typologique des sculptures architecturales de l'abbatiale, qui pourra être mise en parallèle avec les résultats fournis par l'analyse archéologique du bâti.

À ce jour, un premier essai de restitution des arcades de la galerie septentrionale du cloître, à partir des fragments, peut être proposé. Bien que largement hypothétique ${ }^{8}$, il prend en compte l'inventaire des éléments conservés au musée municipal, réalisé en parallèle à l'étude du mobilier issu des fouilles (fig. 6). 
Fig. 6 - Hypothèse d'anastylose de la galerie nord du cloître de Saint-Gilles (relevé Heike Hansen, CAO M.-P. Bonetti).

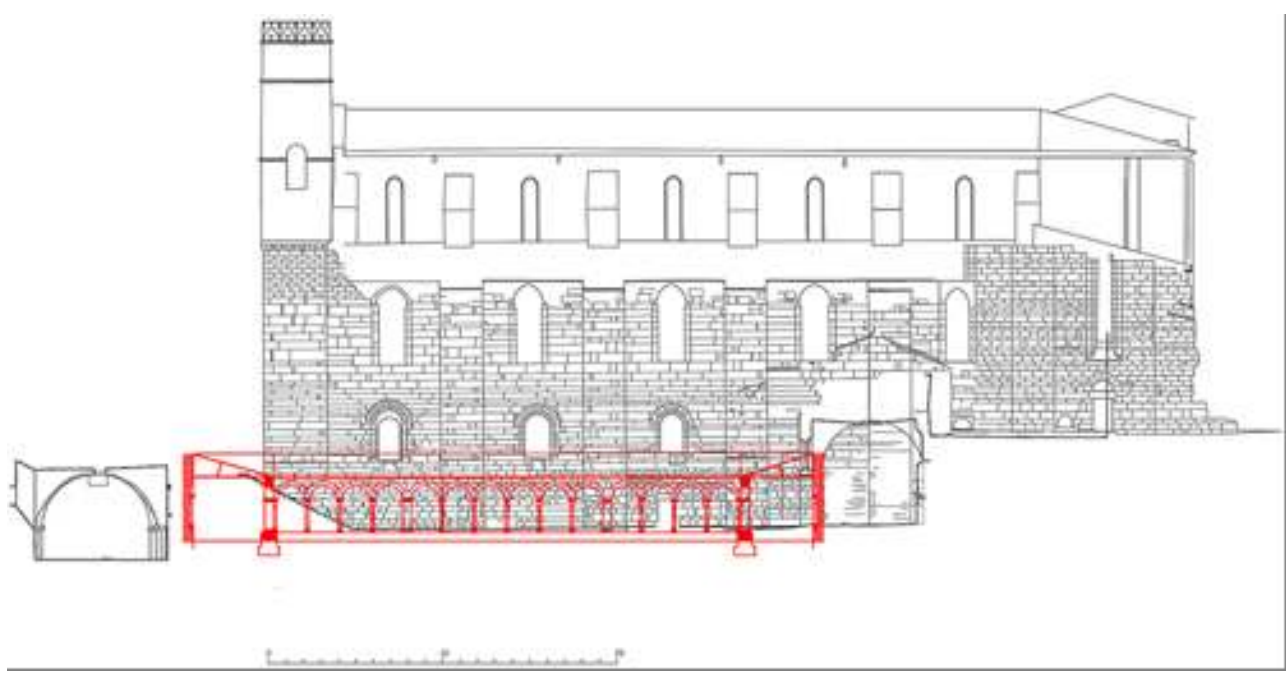

17 Cette collection riche ${ }^{9}$, presque inédite, contient, entre autres, une série de vingt-et-un fûts de colonnette, dont onze cylindriques du même type et calibre que ceux découvertes en fouille, ainsi qu'un ensemble de treize chapiteaux, dont cinq présentent des caractéristiques morphologiques et stylistiques nettement comparables au fragment de corbeille mis au jour en fouille. Leur attribution au cloître ${ }^{10}$ et, à plus forte raison, à une galerie spécifique de ce dernier, reste toutefois conjecturale, d'autant qu'il est incertain si la galerie nord, plus tardive que les autres, fut effectivement dotée d'un nouveau décor lors de sa reconstruction ou simplement remontée ${ }^{11}$. Outre les dimensions fluctuantes des chapiteaux en question ${ }^{12}$, la hauteur des colonnettes reste inconnue en l'absence de fût complet de provenance certaine, tout autant que celle du mur-bahut, dont on ne connait que la largeur de $120 \mathrm{~cm}$ à la base des fondations, d'après le négatif identifié en fouille ${ }^{13}$. Et si la largeur d'arcade calculée à partir de la douelle du sommier est de $162,87 \mathrm{~cm}$, l'analyse de l'ensemble des données devra attendre une étude métrologique des cloitres romans de la région, pour mieux cerner les dimensions comparables.

À cet égard, une restitution de l'architecture de la galerie nord se heurte à d'autres difficultés : la restitution d'un pilastre rectangulaire, forme atypique pour un support libre d'une largeur aussi faible, devrait alors compter avec la présence des deux tailloirs carrés (fig. 7), adaptés soit à un pilier, soit à quatre colonnettes jointives, les deux types de tailloir attestant pour le moins une forme d'alternance des supports et une disposition rythmée des arcades.

Fig. 7 - Tailloir carré provenant du cloître remployé dans l'église inférieure (cl. M.-P. Bonetti).

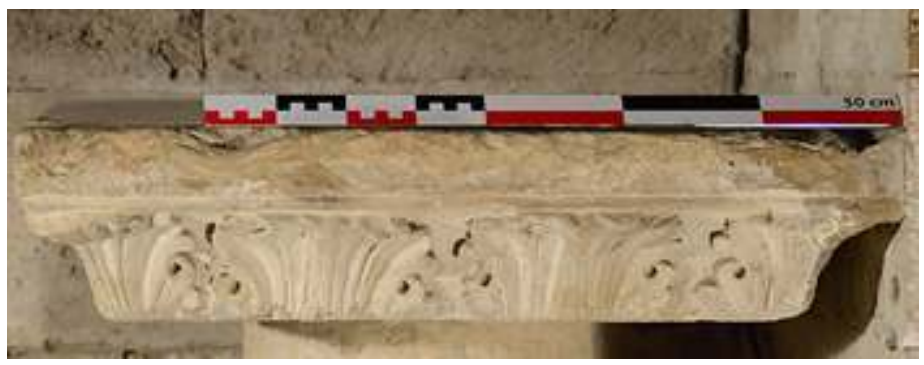



de l'église permettent d'en restituer la hauteur, leur aspect, la hauteur et la forme des toitures, dont la couverture était encastrée dans une rainure ménagée dans le parement du mur gouttereau de l'église, sont incertains.

Si composition architecturale de la galerie septentrionale du cloître de Saint-Gilles n'est pas encore précisément déterminée, le rapprochement stylistique des fragments supposés de son décor avec la sculpture de la façade et du chevet de l'abbatiale est en revanche indiscutable. Le décor végétal de différents éléments sculptés de l'église privilégie les mêmes feuilles d'acanthe charnues, utilisées tant dans la partie méridionale de la frise végétale de la façade que pour les éléments issus des fouilles du cloitre, ainsi que sur le tailloir d'un des chapiteaux de la nef, à la face sud du pilier nord-ouest de l'ancienne croisée, ou encore sur le dernier contrefort oriental situé sur le mur extérieur du bras sud du transept de l'abbatiale. Significatif d'une cohérence stylistique de la sculpture architecturale dans la zone méridionale de l'abbatiale, l'emploi de ce type de feuillage relève de la même période de travaux, de la fin du XII et du début du XIII ${ }^{e}$ siècle, mise en évidence par l'étude archéologique de l'édifice. Par contre, le contexte original des rares fragments de sculpture figurative (fig. 8), qui témoignent de la même proximité stylistique avec la façade de l'abbatiale, comme avec la façade et le cloître de Saint-Trophime d'Arles, reste encore difficile à appréhender.

Fig. 8 - Fragment de personnage en haut-relief mis au jour en fouille (cl. M.-P. Bonetti).

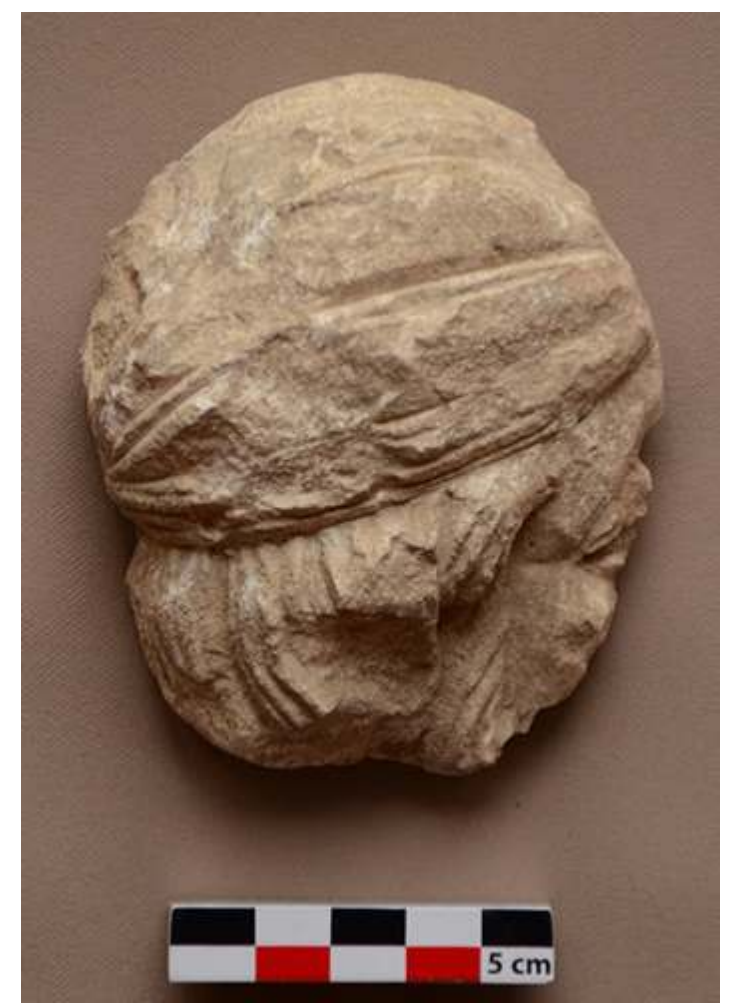

Bien que les éléments retrouvés pour l'instant ne permettent aucune hypothèse précise, leur finesse accuse une fois de plus l'ampleur et la qualité du programme de sculpture architecturale qui fut réalisé à la fois pour la façade, le chevet et les parties du cloître rebâties en même temps, en compensant l'espace claustral sacrifié par une parure monumentale inédite, à la hauteur des exigences de l'époque de 
l'épanouissement du style antiquisant dans l'architecture romane méridionale. Ces découvertes archéologiques récentes, si fragmentaires soient-elles, procurent des indices essentiels pour une relecture du complexe abbatial saint-gillois. Elles appellent à un véritable renouvellement des méthodes d'étude de la sculpture monumentale et, notamment, à un examen systématique de la sculpture erratique, trop souvent reléguée dans les réserves d'un musée ou d'un dépôt lapidaire, car elle peut se révéler un outil décisif pour la compréhension et la restitution de zones entières d'un édifice disparu, comme le cloître de Saint-Gilles-du-Gard. Il s'agit donc de relever un défi scientifique pour accomplir un devoir patrimonial en permettant au public de resituer les vestiges d'une histoire monumentale fragmentaire et méconnue dans leur contexte perdu.

Reçu : 4 mars 2013 - Accepté : 3 mai 2013

\section{NOTES}

1. «AEGIDIANA - L'abbaye de Saint-Gilles-du-Gard. Recherches archéologiques, d'archéologie du bâti et archivistiques sur l'histoire monumentale d'un chef-d'œuvre oublié de l'art roman en France", financé par l'Agence nationale de la recherche (ANR) et la Deutsche Forschungsgemeinschaft (DFG), codirigé par A. Hartmann-Virnich, K.-J. Philipp et H. Hansen, a fédéré le Laboratoire d'archéologie médiévale Méditerranéenne (LAMM - UMR 6572) Université de Provence/CNRS et l'Institut für Architekturgeschichte (IFAG) de l'université de Stuttgart.

2. A. HARTMANN-VIRNICH et M.-P. BONETTI, «Les fragments de sculpture architecturale issus des fouilles du cloître de l'ancienne abbaye de Saint-Gilles-du-Gard", in A. HARTMANN-VIRNICH et H. HANSEN (dir.), L'ancienne abbaye de Saint-Gilles-du-Gard. Nouvelles recherches archéologiques sur un monument majeur de l'art roman, Paris, 2013 (éd. Bulletin monumental, $\mathrm{n}^{\circ}$ 3, 2013), à paraître.

3. A. HARTMANN-VIRNICH et H. HANSEN, «L'église abbatiale et les bâtiments monastiques : nouvelles recherches archéologiques ", in A. HARTMANN-VIRNICH et H. HANSEN (dir.), L'ancienne abbaye..., ibid. ; A. HARTMANN-VIRNICH, L. BUfFAT, L. SCHNEIDER, A. LEGRAND-GARNOTEL et A. MASBERNAT-BUfFAT, avec la collaboration de H. HANSEN et C. MARKIEWICZ, "L'ancienne abbaye de Saint-Gilles-du-Gard: les vestiges architecturaux du sous-sol d'après les investigations archéologiques ", in A. HARTMANNVIRNICH et H. HANSEN (dir.), L'ancienne abbaye..., ibid.

4. Cf. A. HARTMANN-VIRNICH, «Catalogue des fragments de décor architectural issu des fouilles », in A. MASBERNAT-BUFFAT, L. BUFFAT, A. HARTMANN-VIRNICH, A. LEGRAND-GARNOTEL, L. SCHNEIDER et M.A. CHAZOTTES, Le cloitre de l'abbatiale. Saint-Gilles-du-Gard (30). Rapport de fouille, SRA, Montpellier, 2011, p. 203-222.

5. Il convient de rappeler le verdict de Willibald Sauerländer, qui dénonçait, dès 1979, l'intérêt «tout au plus nombrilique » de ce type d'étude à l'exemple de l'historiographie de la façade de Saint-Gilles, cf. W. SAUERLÄNDER, «Das 10. Internationale Kolloquium der Société française d'archéologie: die Fassade der Abteikirche in Saint-Gilles-du-Gard », Kunstchronik, 31 (1978), p. 45-55, p. 54-55. A. HARTMANN-VIRNICH, avec la collaboration de H. HANSEN, «La façade de l'abbatiale de Saint-Gilles-du-Gard", in Congrès archéologique de France, $157^{\circ}$ session, Gard, 1999, Paris, 2000, p. 271-292, ici p. 287-288; ID., «Sur les traces des sculpteurs et maçons. Les découvertes archéologiques ", in A. HARTMANN-VIRNICH (dir.), Le portail de Saint-Trophime d'Arles. Naissance et renaissance d'un chef-d'œuvre roman, Arles, 1999, p.107-157, ici p.121-132 ; ID., « Du 
programme décoratif à la mise en œuvre. Les chapiteaux du portail et de la galerie nord du cloître de Saint-Trophime d'Arles", in Apocalypse, visions et représentations à l'époque romane. Recherches récentes sur l'art roman, éd. Revue d'Auvergne, 116 (2002), p. 33-71, ici p. 35-36 et 51-64 ; ID., «Les galeries romanes du cloitre de Saint-Trophime d'Arles: études sur un chantier de prestige ", in P. K. KLEIN (dir.), Der mittelalterliche Kreuzgang - The Medieval Cloister - Le cloitre au Moyen Age. Architektur, Funktion und Programm, Ratisbonne, 2004, p. 285-316, p. 297-298.

6. « AEGIMAIOR - L'Ancienne abbaye de Saint-Gilles-du-Gard. Archéologie et histoire monumentale d'un site monastique majeur du Midi de la France », sous direction d'A. HartmannVirnich.

7. Cf. M.-P. BONETTI, La sculpture architecturale de l'abbaye de Saint-Gilles-du-Gard (30). Inventaire, archéologie, méthodologie, mémoire de master 1 en archéologie et histoire de l'art, sous la direction d'Andreas HARTMANN-VIRNICH, université d'Aix-Marseille I, 2012, 4 vol.

8. Le relevé manuel à l'échelle 1:1 de l'écoinçon et du sommier ont permis de procéder à une restitution sous AutoCAD. Cependant, l'état de conservation très fragmentaire de la corbeille de chapiteau, des fûts de colonnes ainsi que des bases ne permettait pas de recréer toutes les composantes d'une « arcade-type ».

9. L'inventaire du musée de la Maison romane de Saint-Gilles réalisé en 2012 contient 184 pièces, dont la très grande majorité $\mathrm{du} \mathrm{XII}^{\mathrm{e}}$ siècle provient sans doute de l'abbatiale, notamment des parties hautes du chevet détruit au XVIII ${ }^{\mathrm{e}}$ siècle, cf. M.-P. BONETTI, La sculpture architecturale..., op. cit., 2012 (t. II-IV, inventaire du musée intitulé MUSLAP).

10. L'origine des chapiteaux étant incertaine, on ne peut exclure une provenance d'autres édifices religieux ou civils romans, dont le décor in situ accuse une parenté avec le chantier abbatial.

11. La tranchée d'épierrement de la galerie nord du cloître mise en évidence par la fouille indique que celle-ci a été construite par glissement au sud, suite à un rétrécissement de l'espace claustral lors des travaux d'agrandissement et reconstruction de l'abbatiale dans le derniers tiers du XII ${ }^{\mathrm{e}}$ siècle, cf. L. BUFFAT, «Les éléments bâtis du cloître : mise en place et modifications», in A. MASBERNAT-BUFFAT et alii, Le cloître de l'abbatiale..., op. cit, p. 73-80 et 95-100.

12. Chapiteaux : hauteur $30,6 \mathrm{~cm} \times 21,25 \mathrm{~cm}$ de largeur maximale et $15,5 \mathrm{~cm}$ de largeur au lit de pose ; fûts: diamètre $14,6 \mathrm{~cm}$; bases avec plinthe: hauteur $23,9 \times 29,2 / 26,5 \mathrm{~cm}$ de largeur ; tailloir : hauteur $14,3 \mathrm{~cm} \mathrm{x} 64 / 48 \mathrm{~cm}$, respectivement $64 / 62,5 \mathrm{~cm}$. Les dimensions se fondent sur une moyenne statistique des deux seuls chapiteaux sculptés sur les quatre faces conservés au musée, ainsi que des fûts et fragments de fût conservés au musée et issus de la fouille du cloître. La base utilisée pour cette reconstitution est le seul élément de ce type conservé dans son intégralité, cependant il est impossible d'affirmer son appartenance à la galerie nord. Outre le tailloir rectangulaire trouvé en fouille, deux autres tailloirs attribuables au même contexte, l'un remonté dans le collatéral sud de la crypte sur un fragment de fût de colonne et l'autre déposé au musée, sont de forme carrée.

13. Elle est aussi sans rapport certain avec la hauteur du soubassement de l'arcade latérale au sud. 
INDEX

Mots-clés : abbaye, sculpture, Aegidiana, méthodologie, Aegimaior, inventaire, fouilles, musée de la Maison romane

Index géographique : France/Saint-Gilles-du-Gard

\section{AUTEURS}

ANDREAS HARTMANN-VIRNICH

Université d'Aix-Marseille I, LA3M (UMR 7298)

\section{MARIE-PIERRE BONETTI}

Université d'Aix-Marseille I, LA3M (UMR 7298) 